intramuscular gentamicin in adults, for any indication, in studies where a comparator was available.

Methods A review protocol was developed and registered (PROSPERO: CRD42013003229). Studies were eligible for review if they; recruited participants $\geq 16$ years old, used gentamicin intramuscularly or intravenously as a single one-off dose, compared gentamicin to another medication or placebo, and if adverse events were monitored. We searched MEDLINE, EMBASE and other relevant databases. Risk of bias was assessed in included studies.

Results 12,116 records were identified. After removal of duplicates, screening of title/abstracts for relevance and independent selection of full texts by two reviewers, 20 studies were included. 3589 participants were analysed across all studies, 2042 received a single one-off dose of gentamicin (doses ranged from $1 \mathrm{mg} / \mathrm{kg}$ - $280 \mathrm{mg}$ ). Reversible nephrotoxicity/creatinine rise was reported in 37 cases, with one case of irreversible renal impairment. There were three cases of ototoxicity, with similar frequency reported in the comparator group. A meta-analysis was not possible due to heterogeneity. Reporting of adverse events was poor in the majority of studies.

Discussion Adverse events with single dose gentamicin are infrequent.

\section{P062 A RETROSPECTIVE AUDIT OF HEPATITIS B MANAGEMENT IN THE GU CLINIC}

Elizabeth Nicol*, Chris Ward. The Hathersage Integrated Contraception, Sexual Health and HIV service, Manchester, UK

\subsection{6/sextrans-2016-052718.116}

Background/introduction Background/introduction: Hepatitis B (HBV) is a sexually transmitted infection commonly diagnosed in GU settings. We routinely test for $\mathrm{HBV}$ in high risk patients such as men who have sex with men, sex workers and those from high prevalence areas.

Aim(s)/objectives A retrospective audit was undertaken assessing whether patients diagnosed with $\mathrm{HBV}$ are being managed in accordance with BASHH guidance and whether changes made following a previous audit were implemented successfully.

Methods Patients newly diagnosed with HBV over an 18 month period up to $31 / 8 / 15$ had their records reviewed. Data was collected on demographics, investigations, initial management and follow up.

Results 31 patients were included in the audit and their care compared to the 2008 national auditable standards. Median age was 28(range 16-46). 20 (64.5\%) were male and $11(35.5 \%)$ female. $29(93.5 \%)$ identified as heterosexual, $2(6.5 \%)$ as homosexual. 11 (35.5\%) were of African descent. Only 6\% of patients were provided with written information on HBV transmission and outcomes and 68\% had documented partner notification. 95\% had liver function tests performed post diagnosis, $97 \%$ had clear long term management plans documented and $87 \%$ were offered appointments with hepatology(compared to $67 \%$ in the previous audit).

Conclusion Clinicians were failing to provide written information about HBV following diagnosis as information leaflets were not available in clinic. $87 \%$ of patients received verbal health advice but this still falls short of national standards. A scheme giving cards with QR codes linking to approved patient information is being trialled. Improved referral pathways have resulted in more patients having specialist review.

\section{P063 STAFF, ASSOCIATE SPECIALIST AND SPECIALTY (SAS) DOCTORS' NATIONAL AUDIT ON THE MANAGEMENT OF GONORRHOEA IN THE UK}

${ }^{1}$ Helen Mullan*, ${ }^{2}$ Carlos Oroz, ${ }^{3}$ Jane Richards, ${ }^{4}$ John Lee. ${ }^{1}$ Central London Community Healthcare NHS Trust, London, UK; ${ }^{2}$ Chalmers Sexual Health Centre, Edinburgh, UK; ${ }^{3}$ Newcastle Hospitals NHS Foundation Trust, Newcastle-upon-Tyne, UK; ${ }^{4}$ Josephine Butler Centre, Wakefield, UK

\subsection{6/sextrans-2016-052718.117}

Background/introduction The British Society for Sexual Health and HIV (BASHH) revised United Kingdom national guideline for the management of gonorrhoea in adults, 2011, identified five auditable outcome measures. The UK National Guideline for Gonorrhoea Testing, Clinical Effectiveness Group, BASHH, 2012, suggested a further three.

Aim(s)/objectives The aim was to audit national management of gonorrhoea against the standards recommended in these two documents. Only SAS doctors were eligible to participate.

Methods SAS doctors, whose details were registered on the SAS database, were invited to contribute. All clinics were asked to designate a Local Co-ordinator who would register that clinic and allocate patients to the other participating doctors. Results for the individual clinics were sent to the Local Co-ordinator for dissemination. All information was submitted via a secure online link. Data from forty patients was requested.

Results 3233 cases were submitted from 78 centres by 168 doctors. $68 \%$ cases were male and 44\% were MSM. $83 \%$ received first line treatment. $97 \%$ were tested or treated for CT. $41 \%$ offered written information on GC. Culture attempted in $86 \%$ of those GC NAAT positive. Sensitivity testing performed on 94\% culture positive. Supplementary testing performed on $61 \%$ throat and $60 \%$ rectal NAAT reactive. TOC performed on $61 \%$; $41 \%$ of these within 2 weeks. Partner notification was done in 92\% cases.

Discussion/conclusion $83 \%$ patients received first-line treatment. A reason was provided for $11 \%$ treated with other regimens. The number offered written information was low at $41 \%$. Results about supplementary testing were inconsistent. The results for the other outcomes were satisfactory.

\section{P064 DELIVERY OF SEXUAL HEALTH INTERVENTIONS FOR MEN WHO HAVE SEX WITH MEN (HPV VACCINATION AND PREP) MAY BE HINDERED BY THE LACK OF SEXUALITY DISCLOSURE IN PRIMARY CARE}

${ }^{1}$ Samuel Owen*, 1,2Thomas Kurka, 1,2Daniel Richardson. 'Brighton and Sussex Medical School, Brighton, UK; ${ }^{2}$ Brighton \& Sussex University Hospitals NHS Trust, Brighton, UK

\subsection{6/sextrans-2016-052718.118}

Background/introduction Large scale new sexual heath interventions for MSM (HPV vaccination, PrEP) will potentially need to be delivered in primary care as well as sexual health. It is important these services are acceptable to MSM and MSM feel confident to disclose their sexuality.

Methods From November 2015 to February 2016, a paper survey was distributed to MSM attending local sexual health services on acceptability of local services and initial disclosure of sexuality to a healthcare setting. National Student Pride also used an online version of the survey.

Results 1186 MSM were included in the analysis of this survey. The median age was 26.8 years (18-89). 1026/1186 (86.5\%) self-identified as gay, 108/1186 (9.1\%) bisexual and 34/1186 
(2.9\%) straight. 918/1186 (77.4\%) were HIV-negative, 42/1186 (3.5\%) HIV-positive, 188/1186 (15.9\%) never tested, and 38/ 1186 (3.2\%) unknown status. The median age of first sexual behaviour with another man was 17.6 years (6-41). The median age of disclosure of sexuality to: family is 18 years $(8-45)$ and friends 17 years (11-41); sexual health services 892/1186 $(66.6 \%)=19.0$ years $(14-54)$; and to primary care $522 / 1186$ $(44 \%)=21.2$ years $(13-54)$. There is a difference between age of first sexual experience and disclosure to primary care of 3.6 years.

Discussion/conclusion Delayed disclosure to healthcare professionals of sexuality by MSM is likely to impede the uptake of important health interventions in MSM.

\section{P065 LACK OF EXPOSURE TO GENITOURINARY MEDICINE (GUM) IS LEADING TO A RECRUITMENT CRISIS}

${ }^{1,3}$ Anna Hartley ${ }^{*},{ }^{2-4}$ Daniel Richardson. ${ }^{1}$ Barts Health NHS Trust, London, UK; ${ }^{2}$ Brighton \& Sussex University NHS Trust, Brighton, UK; ${ }^{3}$ British Association for Sexual Health and HIV; ${ }^{4}$ Brighton \& Sussex Medical School, Sussex, UK

\subsection{6/sextrans-2016-052718.119}

Background In 2015, genitourinary medicine (GUM) filled 46\% of its national training numbers. Reasons for low recruitment are unclear. In February, GUM exhibited at the Royal College of Physicians (RCP) Medical Careers Day attended by undergraduates (UGs) and junior doctors (JDs).

Aim We aimed to assess the factors that attract and deter delegates from choosing a career in GUM.

Methods A survey was conducted amongst delegates who visited the GUM stall at the Careers Day.

Results 93\% (25/27) of delegates who visited the stall completed the survey (14 UGs, 8 foundation year (FY) doctors, 3 other). $33 \%(8 / 24)$ would like a career in GUM (54\% (13/24) not sure; $13 \%(3 / 24)$ were not interested in GUM). 92\% (23/25) would like/have liked a rotation in GUM as a JD. 76\% (19/25) were exposed to GUM in medical school (86\% FYs, 50\% UGS). One delegate had done a rotation in GUM as a FY. The table shows the main factors that attract delegates to or deter them from a career in GUM:

\begin{tabular}{llll}
\multicolumn{2}{l}{ Abstract P065 Table 1 } \\
\hline Attracts & $\begin{array}{l}\text { Number of } \\
\text { delegates }\end{array}$ & Deters & $\begin{array}{l}\text { Number of } \\
\text { delegates }\end{array}$ \\
\hline $\begin{array}{l}\text { "Variety/interesting } \\
\text { speciality" }\end{array}$ & 11 & $\begin{array}{l}\text { Lack of exposure to } \\
\text { GUM }\end{array}$ & 5 \\
Work-life balance & 7 & $\begin{array}{l}\text { Lack of inpatient } \\
\text { work }\end{array}$ & 3 \\
Research opportunities & 4 & & \\
HIV & 4 & & \\
\hline
\end{tabular}

Conclusion This survey shows that there is interest in GUM at UG/JD level. A variety of factors appealed to delegates, with fewer deterrents, of which "lack of exposure" predominated. Delegates would like GUM rotations as JDs. Optimising exposure to GUM within medical schools and JD rotations should be a priority in order to attract trainees to GUM.
P066 TRANSMISSION OF NEISSERIA GONORRHOEAE AMONG MEN WHO HAVE SEX WITH MEN: AN ANATOMICAL SITE-SPECIFIC MATHEMATICAL MODEL AND IMPACT OF MOUTHWASH

1,2 Lei Zhang ${ }^{*}$, ${ }^{3}$ David Regan, ${ }^{1,2}$ Eric Chow, ${ }^{1,2}$ Vincent Cornelisse, ${ }^{5}$ Manoj Gambhir, 1,2Jane Hocking, ${ }^{1}$ Christopher Fairley. ${ }^{1}$ Melbourne Sexual Health Centre, Melbourne, Australia; ${ }^{2}$ Central Clinical School, Faculty of Medicine, Monash University, Melbourne, Australia; ${ }^{3}$ Kirby Institute, Faculty of Medicine, University of New South Wales, Sydney, Australia; ${ }^{4}$ Melbourne School of Population and Global Health, University of Melbourne, Melbourne, Australia; ${ }^{5}$ School of Public Health and Preventive Medicine, Faculty of Medicine, Monash University, Melbourne, Australia

\subsection{6/sextrans-2016-052718.120}

Background/introduction Epidemiological data suggest that kissing may play a significant role in gonorrhoea transmission.

Aim(s)/objectives We developed a transmission model to explain anatomical site-specific prevalence of gonorrhoea among Australian men who have sex with men (MSM) and evaluate the population-level impacts of screening and the use of mouthwash as interventions in reducing its transmission.

Methods We constructed a gonorrhoea transmission model to estimate the per-act transmission probability. Using Monte-Carlo simulations, we constructed hypothetical scenarios to evaluate its population-level impacts.

Results We have previously reported the prevalence of pharyngeal, anal and urethral gonorrhoea as being 10.6\% (95\%CI 8.1$12.2 \%), 8.6 \%(6.7-10.4 \%)$ and $0.17 \%(0.02-0.24 \%)$, respectively, in Australian MSM. Calibrated to these data, the modelestimated per-act transmission probability for gonorrhoea was high for transmission from urethra-to-anus (46.0\% [41.752.6\%]) and from-urethra to-pharynx (49.6\% [46.7-53.8\%]). Although pharynx-to-pharynx transmission through kissing has only a transmission probability of $17.4 \%$ (16.0-21.0\%), it accounts for nearly three quarters of the annual incident cases (74.6\% [70.0-82.4\%]). A substantial increase in gonorrhoea screening from the current $40 \%$ to $100 \%$ may only halve gonorrhoea prevalence in MSM. In contrast, the use of mouthwash with moderate efficacy (extra 1\% bacterial load reduction/use) would further reduce the corresponding site prevalence to $2.4 \%$ $(1.8 \%-3.7 \%), 2.2 \% \quad(1.6-3.2 \%)$ and $0.02 \% \quad(0.01-0.03 \%)$, whereas a high efficacy (extra $1.5 \%$ reduction/use) may achieve a scenario of close to elimination.

Discussion/conclusion Our results suggests that kissing may be the key driver of community prevalence. If antibacterial mouthwash is effective and widely used, it may contribute to controlling the gonorrhoea epidemic.

\section{P067 WHY DON'T PEOPLE WITH GENITO-URINARY SYMPTOMS GO TO SEXUAL HEALTH CLINICS? A MIXED METHODS STUDY ABOUT MEANINGS OF SYMPTOMS AND CARE-SEEKING USING THE THIRD NATIONAL SURVEY OF SEXUAL ATTITUDES AND LIFESTYLES (NATSAL-3)}

${ }^{1}$ Fiona Mapp*, ${ }^{1}$ Ford Hickson, ${ }^{2}$ Cath Mercer, ${ }^{1}$ Kaye Wellings. 'London School of Hygiene \& Tropical Medicine, London, UK; ${ }^{2}$ University College London, London, UK

\subsection{6/sextrans-2016-052718.121}

Background/introduction There are both individual and public health benefits in people responding to genito-urinary symptoms effectively. Sexual health clinics are best equipped for managing symptoms but not everyone with symptoms chooses to attend. 